\title{
Article
}

\section{Effects of exercise interventions on physical function, mobility, frailty status and strength in the pre-frail population: A review of the evidence base for practice}

Roddam, Hazel, Stewart, Heather Christine and Lewis, Felicity Available at http://clok.uclan.ac.uk/29218/

Roddam, Hazel ORCID: 0000-0002-0637-1801, Stewart, Heather Christine ORCID: 0000-0002-4657-3221 and Lewis, Felicity (2021) Effects of exercise interventions on physical function, mobility, frailty status and strength in the pre-frail population: A review of the evidence base for practice. European Journal of Physiotherapy, 23 (2). pp. 86-94. ISSN 2167-9169

It is advisable to refer to the publisher's version if you intend to cite from the work. http://dx.doi.org/10.1080/21679169.2019.1645882

For more information about UCLan's research in this area go to

http://www.uclan.ac.uk/researchgroups/ and search for < name of research Group>.

For information about Research generally at UCLan please go to http://www.uclan.ac.uk/research/

All outputs in CLoK are protected by Intellectual Property Rights law, including Copyright law. Copyright, IPR and Moral Rights for the works on this site are retained by the individual authors and/or other copyright owners. Terms and conditions for use of this material are defined in the policies page. 
Short title: Pre-Frail Exercise

$3 \quad$ Full title:

4 Effects of exercise interventions on physical function, mobility, frailty status and strength

5 in the pre-frail population: A review of the evidence base for practice

6 Felicity J. Lewis ${ }^{\mathrm{ab}}$, Heather C. Stewart ${ }^{\mathrm{b}}$, Hazel Roddam ${ }^{\mathrm{b}}$

7 aLancashire Care NHS Foundation Trust, Community Emergency Response Team Southport 8 and Formby, United Kingdom.

$9{ }^{b}$ School of Health Sciences, University of Central Lancashire, PR1 2HE, United Kingdom.

$11{ }^{*}$ Correspondence: Dr Hazel Roddam, School of Health Sciences, University of Central

12 Lancashire PR1 2HE Email: $\underline{\text { HRoddam@uclan.ac.uk }}$

14 Word Count:

Structured Abstract: 190

Main Body: 2931 

and strength in the pre-frail population: A review of the evidence base for practice

Abstract

Background: Frailty is associated with reduced functional ability. Pre-frail individuals are at increased risk of becoming frail and are more likely to transition back to a robust state than frail individuals. Exercise has been reported to have beneficial effects on physical function in combined pre-frail and frail populations. This review identified the need to investigate the pre-frail population in isolation.

Objectives: To investigate the effects of exercise interventions on physical function, mobility, frailty status and strength in the pre-frail population, and to support the role of physiotherapy in the management of pre-frailty.

Data Sources: The electronic databases AMED, CINAHL Complete, MEDLINE with Full Text and PubMed were searched using terms related to pre-frailty, exercise, strength, mobility and function.

Results: The search yielded 456 articles. Seven RCTs and two NRSs were eligible and methodological quality varied from good to poor. Interventions included combinations of strengthening, balance, functional, mobility, power and wii-fit exercises. 
Conclusions: Exercise is an effective intervention to improve physical outcomes and potentially delay or reverse frailty in the pre-frail population. Further high quality research is required to support the recommendations made by this review.

40

41 Contribution of the Paper:

- The term pre-frail refers to the state between robust and frail and is associated with an increased risk of becoming frail.

- Exercise interventions can have positive effects on physical function, mobility and strength in the pre-frail population.

- Physiotherapists are well placed to deliver exercise interventions and manage prefrail patients.

- The current evidence base is insufficient; further research of high quality is required to investigate the effects of exercise and early physical exercise intervention in the pre-frail population.

Keywords: Pre-frail, exercise, physical function, mobility, frailty 
Frailty is a dynamic state that refers to a lack of physiological reserve and reflects accelerated aging $[1,2]$. Frailty is also associated with adverse health outcomes resulting in reduced functional ability and high usage of health and social care services in the UK [2-4]. The term pre-frail refers to the state between robust and frail and is associated with an increased risk of becoming frail [5].

Currently the gold standard of care for managing frailty is the provision of a comprehensive geriatric assessment (CGA) [6]. CGAs are carried out by medical and allied health professionals (AHPs) with physiotherapists assessing key aspects of frailty such as physical function, mobility, strength and balance [7]. Following a CGA it is recommended that an individualised multi-disciplinary intervention plan is developed [7], which physiotherapists play a key role in delivering [8]. This is supported by Professor Hobbelen, a leading health researcher who at a 2016 European Region - World Confederation for Physical Therapy conference described physiotherapists as possessing the "golden bullet" of exercise to fight frailty [9].

Several reviews support the potential of exercise as an effective intervention to improve physical outcomes in frail and combined pre-frail and frail populations [11-13]. A recent systematic review investigating the effects of health promotion in the pre-frail population reported improvements in physical function with exercise [10]. However, the search terms did not include those relating to exercise and included studies investigating combined preand moderately frail populations. 
79 Clinically observed differences suggest there is a need for the pre-frail population to be

80

\section{Objectives}

studied in isolation. During a recent 12-month Frailty Clinic pilot at a North West NHS Trust pre-frail patients (per the Rockwood Scale [14]) were more able to participate in physical rehabilitation than frail patients. Additionally, these pre-frail patients demonstrated greater improvements in physical function and mobility.

It was reported by Gill et al. that older people are more likely to transition to greater rather than lesser states of frailty over a prolonged period of time (54-months) [5]. It was also reported that the probability of transitioning back to a robust state from pre-frail and frail states was between $9.5-16.5 \%$ and $0-0.9 \%$ respectively [5]. These findings along with clinical observations suggest that the pre-frail population exist as a key group to target exercise interventions aimed at managing, delaying and reversing frailty.

The aim of this review was to investigate the effects of exercise interventions on physical function, mobility, frailty status and strength in the pre-frail population. The current evidence base was systematically reviewed to determine if any clinical recommendations could be made. The secondary aim was to support the role of physiotherapy in the management of pre-frail patients. 
99

100

101

102

103

104

105

106

107

108

109

110

111

112

113

114

115

116

117

118

\section{Methods}

This review is reported according to the preferred reporting items for systematic reviews and meta-analyses (PRISMA) guidelines [15]. The review question was built on the participants, interventions, comparisons, outcomes and study design (PICOS) framework. The following methodology was carried out by one author.

\section{Search Strategy and Selection Criteria}

Eligible studies were identified by searching the electronic databases AMED, CINAHL Complete, MEDLINE with Full Text and PubMed (last accessed December 2017). Search terms included terms related to pre-frailty, exercise, strength, mobility and function. The search was supplemented by reference list searching of eligible study reports and relevant reviews. Due to the low yield of articles relating specifically to the pre-frail population no limiters were set for date range or study type.

The titles and abstracts identified by the search were reviewed and the full texts of potentially eligible studies were evaluated against the following criteria:

Inclusion Criteria:

- Use of a recognised and referenced frailty tool to classify people as being pre-frail.

- Analysis of pre-frail people in isolation including sub-group analysis. 
- Analysis of exercise as a single intervention compared to a control or comparator group.

- Use of outcome measures that relate to physical function, mobility, frailty status or strength.

- Outcome measures performed before and after the intervention period.

- Full text available in the English language.

Exclusion Criteria:

- Analysis of the pre-frail population with a specific health condition e.g. Parkinson's disease.

- Use of outcome measures not relevant to the review question.

\section{Data Extraction}

A number of study characteristics were extracted from the eligible studies using a table to enable consistent recording. To analyse intervention effects within and between group differences in mean outcome scores were recorded. Significance levels and effect sizes were recorded where available. 


\section{Results}

Methodological quality was assessed using the critical appraisal skills programme (CASP) tool for randomised control trials (RCTs) [16].

The search strategy yielded 456 articles ranging from 2001 to 2018; after duplicates were removed 191 articles remained for title and abstract screening. The full text of 16 articles were retrieved, after applying the inclusion and exclusion criteria 10 articles were deemed eligible for inclusion in the review [17-26]. Reasons for exclusion are outlined in the study selection flow diagram (Figure 1).

Two articles reported on the same original study $[19,26]$, one consisted of a follow-up study after a period of de-training [26]. The study was included as the follow-up period was similar to other eligible studies $[21,22]$ and it was deemed clinically relevant to determine the long term effects of exercise interventions. Another two articles [22, 23] reported on different outcomes of the same study and were evaluated together, resulting in 9 studies to be included in the review.

\section{Study Characteristics}

A summary of the study characteristics is presented in Table 1. Two studies were conducted in Brazil $[17,22,23]$, two in Japan $[24,25]$, two in Germany $[19,26]$ and one each in the USA [18], Netherlands [20] and the Republic of Korea [21]. Seven of the studies were RCTs [17- 
$23,26]$ including a follow-up [26] and a pilot [18]. Two studies were non-randomised control trials (NRS) [24, 25]; one was a pilot [25]. Sample size ranged from 23 [18] to 238 [20] and all studies used the Fried frailty phenotype criteria [27] to identify people as being pre-frail.

\section{Intervention Characteristics}

Study methodologies included comparing single exercise programmes to a control $[17,22$, $23]$ or robust comparator group [24, 25], comparing two different exercise programmes to a control $[18-20,26]$ and comparing a single intervention exercise programme to a combined exercise and nutrition intervention (cooking class) to a control [21]. For the latter study, only data relating to the single intervention exercise group and the control were considered in order to meet the inclusion and exclusion criteria.

The exercise interventions included components of strength, balance, mobility and function. One RCT and its follow-up study compared strength and power training $[19,26]$ and one RCT compared exercises to the wii-fit [18]. Progressive exercise programmes were utilised by all of the RCTs whereas the two NRSs did not.

The duration of the exercise sessions ranged from 45 to 90 minutes, 1 to 7 days a week for 10 to 52 weeks. All studies carried out the exercise programme in a supervised group setting except one [25] which investigated an unsupervised daily home-based programme 
following 1-2 instruction sessions. Only one of the studies utilising a group setting reported instructing participants to perform the exercises at home, detail relating to frequency is not given [21]. Three studies followed up the participants ranging from 10 weeks $[22,23]$ to 6 months $[21,26]$ post-intervention. Two studies asked participants not to carry out any of the intervention exercises after the intervention period had ended $[22,23,26]$, it is unclear if this was the case for the third study [21].

The control groups were asked to continue their daily routines and not start new physical activities [18-20, 22, 23, 26], attend lectures on physical activity and nutrition [19, 21] and carry out upper limb and neck stretches and relaxation [17].

Outcome measures utilised included the timed up and go (TUG) [18, 20, 22-25], one-leg balance test (OLB) $[21,24,25]$, performance orientated mobility assessment (POMA) [20] and short physical performance battery (SPPB) $[19,26]$. Lower scores for the TUG and higher scores for the OLB, POMA and SPPB indicate a better performance [28]. Other measures included sit to stand transfers [17, 18, 20], gait speed [17, 20-24], strength [19, 21-26] and self-reported function [18-20, 21, 26].

\section{Methodological Quality}

A summary of the individual risk of bias for the included studies is presented in Table 2. All RCTs reported randomised allocation and was computer generated in those reporting on 
methodology $[17,19-21,26]$. Three studies had concealment of allocation $[17,19,20]$, one stated that it did not [21] and there was inadequate reporting for two studies [18, 22, 23]. Selection bias was deemed to be low risk $[17,19,20]$, high risk [21] and unclear $[18,22,23]$.

It is unclear if drop-outs had any significant impact in five of the studies [17, 18, 21, 24, 25$]$. Two reported no impact with drop-outs $[19,20]$ and one reported reduced statistical power [26]. Only two studies reported intention to treat analysis [19, 20]. Attrition bias was deemed to be low risk [19, 20, 22, 23], medium risk [26], high risk [21, 25] and unclear [17$18,24]$.

In all studies the participants and personnel delivering the intervention were not blinded.

Due to the nature of the interventions this was not deemed to significantly impact methodological quality. The assessors were blinded to allocation in six studies $[17,19-23$, 26], but not in either of the NRSs $[24,25]$ and it was unclear in one study due to inadequate reporting [18]. Detection bias was deemed to be low risk [17, 19-23, 26], high risk [24, 25] and unclear [18].

Eight studies reported comparable groups at baseline, the pilot RCT reported a significantly younger control group [18] and there was inadequate reporting by one study [25]. Overall methodological quality was deemed to be $\operatorname{good}[19,20]$, fair $[17,22,23,26]$, fair-poor [21] and poor $[18,24,25]$. The poor quality studies were limited by inadequate reporting and 

of these studies were interpreted with caution and greater weighting was given to the RCTs.

two were pilots designed to test feasibility and method $[18,25]$. Consequently, the results

\section{Impact of Interventions}

A summary of the individual study results is presented in Table 3. Studies that investigated two different exercise programmes reported comparable intervention effects at postintervention and follow up [18-20, 26].

\section{Physical function}

Significant positive intervention effects were observed for sit to stand $[17,18]$, semi-tandem test [17], step test [17] and the SPPB [19]. Although no longer significant, SPPB scores remained higher than baseline for the intervention groups after a 24-week detraining period [26]. Variable intervention effects were reported for the OLB test $[21,24,25]$. Utilising combined physical outcome scores one RCT reported positive and negative intervention effects in pre-frail and frail sub-groups respectively [20]. No intervention effects were reported for self-reported function or disability [18-21, 26] except for the combined exercise and nutrition group [21].

\section{Mobility}



but, not for a robust comparator group [24]. After a 10 week detraining period one study reported lower than baseline TUG scores but it is unclear if this was significant [22].

Significant positive intervention effects on gait speed were reported [17, 21-24], which remained after a 10-week period of detraining [22]. However, one study reported no effect at post-intervention or 6-month follow-up [21]. Utilising the POMA, one study reported a positive intervention effect and no effect in pre-frail and frail sub-groups respectively [20].

\section{Frailty Status}

Only the pilot NRS [25] reported on frailty status with $23.5 \%$ of the pre-frail group transitioning to a robust state post intervention. No participants transitioned to a frail state.

\section{Muscle Strength}

Significant positive intervention effects on knee extensor strength were reported $[22,23$,

25]. Strength remained greater than baseline after a 10 week period of detraining but it is unclear if this was significant [22]. No significant effect was observed for either strength or power training on general lower limb strength [19]. However, the power training group demonstrated greater than baseline power after a 24-week detraining period [26]. No effect [24] and significant positive effects $[21,25]$ were reported for grip strength, but this was not maintained at 6-month follow-up [21]. 
264 This review supports exercise as an effective intervention to improve physical outcomes in 265 the pre-frail population. Due to the review limitations it is advised that the following be 266 interpreted with caution.

Two comparable RCTs $[19,20]$ reported that exercise carried out for an hour, twice a week for 12 weeks resulted in improvements in physical function in the pre-frail population. In contrast, variable findings have been reported for frail populations $[12,13]$; this supports clinical observations by suggesting that exercise is most effective in the earlier stages of 273 frailty. studies reporting a positive effect $[17,25]$ stated that the balance task being assessed formed part of the exercise programme. The two studies reporting no effect $[21,24]$

278 delivered the intervention once a week in comparison to twice weekly [17] and daily sessions [25]. This suggests that to observe improvements in functional balance, exercise programmes should include the tasks being assessed and be carried out for at least an hour, twice a week. 
Positive intervention effects were reported for sit to stand transfers $[17,18]$. One study reported a smaller intervention effect $(p=.046)$ compared to functional balance $(p<.005, p<$ .001) [17]. However, unlike the assessed balance tasks, sit to stand practice did not form part of the exercise programme. This further supports the inclusion of assessed tasks into exercise programmes and suggests that greater improvements are observed with task repetition.

Consistent with systematic reviews investigating frail populations [11-13], the favourable results above were not reflected in the self-reported measures of function. This is in contrast to patients reviewed in the frailty clinic. Reasons for these opposing findings may be due to differences between research and practice. In the clinical trials the exercise programmes were pre-set and not person-specific. In clinical practice exercise programmes meaningful improvements. Further research that reflects clinical practice is required.

\section{Mobility}

Most studies reported favourable intervention effects on gait speed [17, 22-24] and one study, delivering the intervention less frequently reported no effect [21]. These findings concur with findings for frail populations [11-13] and suggest exercise at a frequency of at least twice a week is required to increase gait speed. 
Of the studies that utilised the TUG $[17,22-25]$ and the POMA [20] all reported positive intervention effects. This is in contrast to research relating to frail populations [11-13], suggesting that the early delivery of exercise interventions is required to gain improvements in functional mobility.

\section{Frailty Status}

One study reported that exercise reversed frailty [25]. Although of poor quality the findings concur with a recent $\mathrm{RCT}(\mathrm{N}=245$, pre-frail $73 \%$, frail $27 \%)$ that reported a reduction in frailty following a 24 week exercise intervention $(p<.01)$ [29]. These combined findings may reflect the favourable effects exercise has on many of the Fried frailty domains and warrants further research.

The effectiveness of exercise on lower limb strength varied despite similar interventions and may reflect differences in measurement and frequency. Using an isokinetic dynamometer $[23,25]$ and a force plate (during sit to stand) [19] positive and no effect were reported respectively. The interventions for the RCTs $[19,23]$ were carried out twice [19] versus three [23] times a week. These findings suggest that an intervention frequency of greater than twice a week is required to increase lower limb strength in a pre-frail population. 
This review addresses a highly relevant and specific clinical question, adding to the growing evidence base relating to the pre-frail population. A transparent and systematic approach was used to identify and appraise the evidence base and the inclusion and exclusion criteria were clearly defined.

This review has several limitations. The literature search, study selection and critique was carried out by one author. At study level, the control and intervention groups were not treated equally. The majority of the controls did not attend groups and some attended health lectures which may have altered their behaviour. Further standardised research is required.

The review is further limited by the inclusion of poor quality studies. Additionally, there is a lack of studies pertaining to the pre-frail population. As a result, firm conclusions cannot be made and it is recommended that the findings be interpreted with caution.

\section{Clinical implications}

The review findings are deemed clinically relevant as the exercise programmes and outcome measures utilised by the studies reflect clinical practice. The findings support the prescribing of progressive exercise programmes that include strength, balance, and functional mobility exercises, delivered in group settings for an hour, two to three times a week, long term. Clinically, without appropriate funding it will be difficult to deliver the 
recommended frequency of the group exercise sessions and offer this as a long term service. Supporting the pre-frail population to take ownership of their own health is therefore of great importance. Strategies to meet this challenge could include educating and motivating the pre-frail population to develop an exercise habit. Physiotherapists can support this by assisting in the development of pre-frail pathways and services. In particular physiotherapists could provide short courses of group exercise sessions in both the acute and community setting, form stronger links with third sector organisations to signpost people to local exercise and physical activity classes and develop joint initiatives with third sector organisations. Physiotherapists could also assist in developing and supporting targeted public health campaigns.

The review findings also suggest that exercise interventions are most effective at the prefrail stage and one study even reported negative intervention effects in a frail sub-group [20]. Physiotherapists are well placed to deliver these early physical exercise interventions and possess the assessment skills to deliver first contact CGAs. This could result in significant cost savings to the National Health Service (NHS) by reducing Geriatricians workloads and potentially delaying and reversing frailty. This review therefore recommends early physical exercise interventions for the pre-frail population, of which physiotherapists can deliver.

\section{Conclusion}


This systematically-conducted review has demonstrated that exercise can have positive effects on physical function, mobility and strength in the pre-frail population. Exercise is also identified as a potentially effective intervention to delay and reverse frailty. This review highlights the potential of physiotherapists to become key members of a multidisciplinary

371 team delivering services to the pre-frail population, such as the delivery of group based 372 exercise classes. However, due to an insufficient evidence base it is advised that the review findings be interpreted with caution. Further high quality research studying both the effects of exercise and early physiotherapy involvement on physical outcomes and frailty in the pre-frail

377 population is recommended.

\section{Declarations of Interest}

The authors confirm they have no conflicts of interest.

As secondary research this study did not require ethical approval.

This research did not receive any specific grant from funding agencies in the public, commercial, or not-for-profit sectors. 
1. Afilalo J, Alexander KP, Mack MJ, Maurer MS, Green P, Allen LA, et al. Frailty assessment in the cardiovascular care of older adults. J Am Coll Cardiol. 2014 03/04;63(8):747-62.

400 2. Clegg A, Young J, Iliffe S, Rikkert MO, Rockwood K. Frailty in elderly people (vol 381, pg 752, 2013). Lancet. 2013 OCT 19;382(9901):1328-.

3. Cornwell J. The care of frail older people with complex needs: time for a revolution. London: King's Fund. 2012. 
4. National Institute for Health and Clinical Excellence. Dementia, disability and frailty in later life - mid-life approaches to delay or prevent onset. Clinical Guideline 16. London: NICE. 2015.

5. Gill TM, Gahbauer EA, Allore HG, Han L. Transitions between frailty states among community-living older persons. Arch Intern Med. 2006;166(4):418-23.

6. British Geriatrics Society and the Royal College of General Practitioners. Fit for Frailty - Part 2: Developing, commissioning and managing services for people living with frailty in community settings. British Geriatrics Society and the Royal College of General Practitioners. 2015.

7. British Geriatrics Society. Fit for Frailty - Part 1: Consensus best practice guidance for the care of older people living in community and outpatient settings. British Geriatrics Society. 2014.

8. British Geriatrics Society. [Online] Available at: http://www.bgs.org.uk/nursepublications/nursing/nursepublications/consultationphysio-and-older-people. [Accessed 30th October 2017].

9. Chartered Society of Physiotherapy. [Online] Available at: http://www.csp.org.uk/news/2016/11/17/er-wcpt-physios-have-golden-bullet-fightfrailty-say-researchers. [Accessed 30th October 2017].

10. Frost R, Belk C, Jovicic A, Ricciardi F, Kharicha K, Gardner B, et al. Health promotion interventions for community-dwelling older people with mild or pre-frailty: a systematic review and meta-analysis. BMC Geriatr. 2017 07/20;17(1):157-.

11. Chou C, Hwang C, Wu Y. Effect of exercise on physical function, daily living activities, and quality of life in the frail older adults: a meta-analysis. Arch Phys Med Rehabil. 2012;93(2):237-44. 
12. de Labra C, Guimaraes-Pinheiro C, Maseda A, Lorenzo T, Millán-Calenti JC. Effects of physical exercise interventions in frail older adults: a systematic review of randomized controlled trials. BMC geriatrics. 2015;15(1):154.

13. Giné-Garriga $M$, Roqué-Fíguls $M$, Coll-Planas L, Sitjà-Rabert $M$, Salvà A. Physical exercise interventions for improving performance-based measures of physical function in community-dwelling, frail older adults: a systematic review and metaanalysis. Arch Phys Med Rehabil. 2014;95(4):753,769. e3.

14. Rockwood K, Song X, MacKnight C, Bergman H, Hogan DB, McDowell I, et al. A global clinical measure of fitness and frailty in elderly people. CMAJ. 2005 Aug 30;173(5):489-95.

15. Moher D, Liberati A, Tetzlaff J, Altman DG, Prisma Group. Preferred reporting items for systematic reviews and meta-analyses: the PRISMA statement. PLoS medicine. 2009;6(7):e1000097.

16. Critical Appraisal Skills Programme. [Online] Available at: CASP Randomised Controlled Trial Checklist. 2017. [Accessed: $2^{\text {nd }}$ November 2017].

17. Arantes PM, Dias JMD, Fonseca FF, Oliveira AM, Oliveira MC, Pereira LS, et al. Effect of a Program Based on Balance Exercises on Gait, Functional Mobility, Fear of Falling, and Falls in Prefrail Older Women: A Randomized Clinical Trial. Topics in Geriatric Rehabilitation. 2015;31(2):113-20.

18. Daniel K. Wii-Hab for Pre-Frail Older Adults. Rehabilitation Nursing. 2012;37(4):195201.

19. Drey M, Zech A, Freiberger E, Bertsch T, Uter W, Sieber CC, et al. Effects of strength training versus power training on physical performance in prefrail communitydwelling older adults. Gerontology. 2012;58(3):197-204. 
20. Faber MJ, Bosscher RJ, Paw, Marijke J Chin A, van Wieringen PC. Effects of exercise programs on falls and mobility in frail and pre-frail older adults: a multicenter randomized controlled trial. Arch Phys Med Rehabil. 2006;87(7):885-96.

21. Kwon J, Yoshida Y, Yoshida H, Kim H, Suzuki T, Lee Y. Effects of a combined physical training and nutrition intervention on physical performance and health-related quality of life in prefrail older women living in the community: a randomized controlled trial. Journal of the American Medical Directors Association. 2015;16(3):263. e1,263. e8.

22. Lustosa LP, Pereira LSM, Coelho FM, Pereira DS, Silva JP, Parentoni AN, et al. Impact of an exercise program on muscular and functional performance and plasma levels of interleukin 6 and soluble receptor tumor necrosis factor in prefrail communitydwelling older women: a randomized controlled trial. Arch Phys Med Rehabil. 2013;94(4):660-6.

23. Lustosa LP, Silva JP, Coelho FM, Pereira DS, Parentoni AN, Pereira LS. Impact of resistance exercise program on functional capacity and muscular strength of knee extensor in pre-frail community-dwelling older women: a randomized crossover trial. Brazilian Journal of Physical Therapy. 2011;15(4):318-24.

24. Sugimoto H, Demura S, Nagasawa Y, Shimomura M. Changes in the physical functions of pre-frail elderly women after participation in a 1-year preventative exercise program. Geriatrics \& gerontology international. 2014;14(4):975-82.

25. Takano E, Teranishi T, Watanabe T, Ohno K, Kitaji S, Sawa S, et al. Differences in the effect of exercise interventions between prefrail older adults and older adults without frailty: A pilot study. GERIATR GERONTOL INT. 2017 09;17(9):1265-9. 
26. Zech A, Drey M, Freiberger E, Hentschke C, Bauer JM, Sieber CC, et al. Residual effects of muscle strength and muscle power training and detraining on physical function in community-dwelling prefrail older adults: a randomized controlled trial. BMC geriatrics. 2012;12(1):68.

27. Fried LP, Tangen CM, Walston J, Newman AB, Hirsch C, Gottdiener J, et al. Frailty in older adults: evidence for a phenotype. The Journals of Gerontology Series A: Biological Sciences and Medical Sciences. 2001;56(3):M146-57.

28. Rehabilitation Measures Database. [Online] Available at: https://www.sralab.org/rehabilitation-measures. Accessed [25 $5^{\text {th }}$ October 2017].

29. Ng TP, Feng L, Nyunt MSZ, Feng L, Niti M, Tan BY, et al. Nutritional, physical, cognitive, and combination interventions and frailty reversal among older adults: a randomized controlled trial. Am J Med. 2015;128(11):1225,1236. e1. 